\title{
Multi-User Selection Diversity for Spread-Spectrum Multi-Carrier Multiple-Access Systems
}

\author{
Petros L. Katsis, Student Member, IEEE, George K. Karagiannidis, Senior Member, IEEE, \\ and Fotini-Niovi Pavlidou, Senior Member, IEEE
}

\begin{abstract}
Recently, Multi-User Selection Diversity (MUSDiv) for single-carrier systems has been under extensive study on account of the enhancement it provides to system performance with minimum feedback requirements. However, its application to multichannel systems is considered straightforward and thus, it has not been thoroughly examined. In this paper, the performance of MUSDiv is investigated when applied to the spread-spectrum multi-carrier multiple-access system, where the scheduling has to be performed for all the available channels and self interference must also be considered. Specifically, based on the absolute and normalized Signal-to-Noise-Ratio (SNR) scheduling algorithms, two algorithms are presented, modified and optimized, so that they can be applied on a subband instead of a single-channel basis. Moreover, we propose a new scheduling scheme which constitutes a trade-off between the previous schemes, concerning fairness and capacity performance. The new algorithms are related to the symbol Signal-to-Noise-plus-Interference-Ratio (SNIR) instead of SNR and two interference models were devised to this end. Closed-form expressions for the system capacity are extracted for each case, which are compared with simulation results. The research is also extended to the case of non-identical user power profiles among the available subcarriers. The channel state information required to utilize multi-user selection diversity is already necessary for the most common combining schemes, so no more feedback is actually needed.
\end{abstract}

Index Terms-Multi-user selection diversity, orthogonal frequency division multiplexing, proportional fair-scheduling, spread-spectrum multi-carrier multiple-access, system capacity.

\section{INTRODUCTION}

D IVERSITY over time, frequency and space has been summoned to deal with the issue of increasing capacity in a wireless fading environment. In the case of multiple users, wherein each user is considered to experience independent fading, there is another form of diversity, known as MultiUser Selection Diversity (MUSDiv). Research in this field was initiated by the work of Knopp and Humblet [1], where it was proven that the uplink system capacity is maximized when the user with the highest instantaneous power is chosen to make full use of the channel in one time slot. Meanwhile, the remaining users are kept waiting until the channel state changes and another user is picked. This scheme is carried out in a TDMA fashion so that the selected user gets to transmit

Paper approved by G. E. Corazza, the Editor for Spread Spectrum of the IEEE Communications Society. Manuscript received February 3, 2006; revised December 19, 2006 and August 23, 2007. This work was performed within the framework of the Satellite Communications Network of Excellence (SatNex) project (IST-507052) and its Phase-II, SatNex-II (IST- 27393), funded by the European Commission (EC) under its FP6 program.

The authors are with the Electrical and Computer Engineering Department, Aristotle University of Thessaloniki, 54124 Thessaloniki, Greece (email:\{pkatsis, geokarag, niovi\}@auth.gr).

Digital Object Identifier 10.1109/TCOMM.2008.050307 for a number of time slots, until another user emerges. In [2] Tse was led to the same conclusions for the downlink case.

The concept of multi-user selection diversity implies the use of perfect channel estimation and a perfect feedback channel to communicate the channel features of each user. It also states that the channel variations should be slow enough to enable channel estimation, but also fast enough to prevent the selected user from monopolizing the channel for a long time. Unlike conventional communication design, fading is considered an advantage to be exploited by the system [3]. Knopp and Humblet [1] seem to agree on this by showing that this scheme can achieve higher capacity than a Gaussian channel.

While the fading of each user is considered independent, it is not necessarily identical, i.e., the average fading power of each user may vary significantly for a number of reasons, such as the distance from the base station. Thus, if MUSDiv is employed, average system capacity will be enhanced, but lowaverage-fading-power users may find it difficult to gain access, leading to unfair treatment [3]. By utilizing this proportional fair scheduling algorithm, the system chooses the user who reaches a relative peak compared to the user's own average channel state, achieving fairness at the expense of reducing system capacity. In [4] there is an improvement by increasing the feedback. Yang and Alouini [5] view the original proportional fair scheduling scheme from a more practical perspective by substituting throughput with Signal-to-NoiseRatio (SNR), yielding the normalized SNR-based scheduling.

In [6] the issue whether multi-user diversity is worth the amount of channel condition feedback was examined. The performance of the algorithms proposed in [5] was investigated considering reduced feedback by allowing each user to compete for access only if the user's channel quality is above an optimal threshold. Several reduced channel feedback variants are presented in [7], while the prevailing scheme introduced by Yang, Alouini and Gesbert is thoroughly analyzed in [8]. Generally, MUSDiv can provide enhanced system capacity with minimum channel feedback.

Viswanath et al. in [3] proposed opportunistic beamforming for wideband channels as well, where frequency selectivity is a reasonable hypothesis. Multi-user diversity can be put to use in multicarrier systems, where the scheduling is performed on a subchannel basis; the user with the best channel conditions for a specific subchannel is selected for access for that particular subchannel. All the aforementioned scheduling algorithms can be easily adapted for a multicarrier system, if the wideband channel is modelled as a set of parallel, 
independent narrow-band subchannels, with every subchannel considered frequency-flat. The amount of feedback will be increased by a number of times equal to the number of available subchannels. Moreover, multi-user diversity can be combined with power/bit allocation techniques in a Orthogonal Frequency Division Multiplexing (OFDM) system [9], while a more practical approach is found in [10], [11].

If the Multicarrier Code Division Multiple Access (MCCDMA) system is considered [12], the allocated bandwidth is divided into $L$ subbands, with each subband comprising $M$ subcarriers, and each user transmits one symbol in every subband. This leads to multiple access interference (MAI), which can be partially overcome by tracking the channel state and utilizing frequency diversity. There is a dual approach for the uplink, known as Spread Spectrum Multicarrier Multiple Access (SS-MC-MA), introduced in [13]. In this scheme the system provides one subband for each user who transmits symbols in the same subband using CDMA, so that there is self-interference instead of MAI. Uplink channel estimation complexity is highly decreased in comparison with MCCDMA, since all the interfering symbols belong to the same user, so they are transmitted in a synchronous manner and experience the same fading.

The goal of this paper is to investigate the combination of frequency diversity and multi-user selective diversity, which exists in a spread-spectrum multicarrier multiple-access system operating on a frequency selective channel. SS-MC-MA is a scheme that assigns each user a set of exclusive subcarriers, according to an FDMA scheme. From this perspective, it is quite clear that if the set of subcarriers is chosen by exploiting multi-user diversity, the outcome is a "dual" diversity scheme. This paper examines MUSDiv algorithms that maximizes the system capacity by selecting the most appropriate user for the entire subband. Thus, the first part of the scheme is equivalent to a wideband channel with MUSDiv, as described above, with the main differences being the use of subbands (or set of subcarriers) instead of single subcarriers, which a specific user is assigned to and the introduction of Signal-to-Noiseplus-Interference-Ratio (SNIR) to replace SNR.

Our work focuses on system capacity performance. Three algorithms are presented in this paper; the first two are extracted from the well-known absolute and normalized SNRbased scheduling algorithms. A third one is introduced which constitutes a balance between the two extremes, by increasing fairness and reducing capacity performance. Each scheduling scheme is examined by employing two different interference modeling approaches, which eventually yield similar system capacity expressions. In the first part, the three algorithms consider the case where each user has identical short-term average fading powers for all the subcarriers. The second part considers the general non-identical subcarrier powers case.

The outline of the paper is organized as follows. In Section II, the system and channel models used throughout the paper are thoroughly analyzed. Sections III to VII delineate the multiuser selection diversity algorithms proposed for SS-MCMA. Section VIII presents the simulation results and compares them with the analytical expression obtained in the previous sections. In Section IX concluding remarks are drawn.

\section{System And Channel Models}

Let us consider a single-cell wide-band wireless communication model focusing on the uplink part between a base station and $K$ users. A total of $N$ narrowband subchannels have been allocated, organized into $L$ sets of $M$ subchannels each, so that $N=L \times M$. Each such set is called a subband. SS-MC-MA is based on OFDM, thus the subcarriers are orthogonal and the time slot length and OFDM subcarrier spacing have been carefully chosen so that the flat fading model can be adopted for each subchannel. However, the entire channel bandwidth is greater than the channel coherence bandwidth, a necessary hypothesis to induce frequency selectivity and enhance frequency diversity gain [14]. In a classical SSMC-MA structure every user would be assigned one subband to transmit its symbols. Depending on the multiple access scheme, the number of subbands would have to be equal to the number of users, otherwise not all users would be able to transmit during the same OFDM symbol. This is not the case here, since subbands are assigned with multi-user selection diversity criteria.

Consequently, each OFDM subcarrier is considered narrowband, so the following simple channel model can be adopted

$$
r_{k n}(t)=h_{k n}(t) x_{n}(t)+n_{k n}(t)
$$

where $x_{n}(t)$ is the complex transmitted signal in time slot $t$ on subcarrier $n, r_{k n}(t)$ is the complex received signal from user $k$ in time slot $t$ on subcarrier $n, h_{k n}(t)$ is the channel gain from user $k$ toward the base station in time slot $t$ on subcarrier $n$, and $n_{k n}(t)$ is an independent, identically distributed sequence of zero mean complex Gaussian noise with variance $\sigma_{n}^{2}$. A quasi-static fading channel model is used, where the $h_{k n}(t)$ gains are independent identically distributed (i.i.d.) random variables (RVs) with respect to subcarrier (subscript $n$ ), but not necessarily identical between different users (subscript $k$ ), though they remain independent and are considered constant over a certain integer number of time slots. The amplitude $a_{k n}(t)$ of $h_{k n}(t)$ is assumed to follow the Rayleigh distribution with probability density function (pdf) given by

$$
f_{a_{k}}(a)=\frac{2 a}{\Omega_{k}} \exp \left(-\frac{a^{2}}{\Omega_{k}}\right), a \geq 0
$$

where $\Omega_{k}=\overline{\left(a_{k}^{2}\right)}$ is the short-term average fading power of the $k$ th user.

SS-MC-MA employs a combiner before the detector to exploit frequency diversity. A user who has been granted access to transmit in a subband can transmit $M$ (full load) or fewer symbols per OFDM symbol in order to preserve code orthogonality and each symbol is multiplied by a unique code and spread over $M$ subcarriers.

SS-MC-MA analysis is identical to MC-CDMA if users and user symbols are swapped. Therefore, following an approach similar to the one in [15], the expanded received signal over 


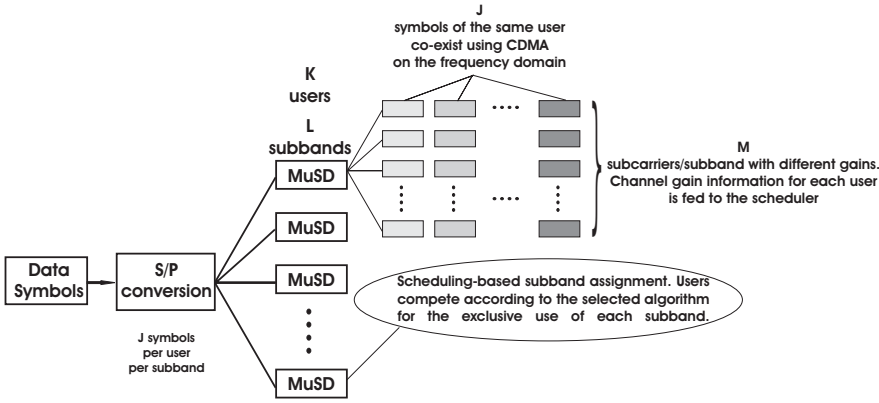

Fig. 1. Block Diagram of the System.

an infinite number of OFDM symbols has the following form

$$
\begin{aligned}
r(t)= & \sqrt{\frac{E_{s}}{M}} \sum_{q=-\infty}^{\infty} \sum_{l=1}^{L} \sum_{j=1}^{J} d_{l j}(q) \\
& \times \sum_{m=1}^{M} a_{l m}(t) c_{l m j}(q) e^{j 2 \pi l m \Delta f\left(t-q T_{s}\right)}+n(t)
\end{aligned}
$$

where $d_{l j}$ is the baseband symbol, $\frac{E_{s}}{M}$ is the power assigned to each baseband symbol, the $j$ index represents the $j$ th symbol transmitted in subband $l$ which can range from 1 to $J$ ( $J=M$ in a fully loaded system), $c_{l m j}$ denotes the orthogonal spreading sequence chip (e.g. by using Hadamard codes [14]) corresponding to the $l$ th subband, $m$ th subcarrier and $j$ th symbol (the $l$ index can be left out, as the users can employ the same spreading sequences, since the spreading is performed inside the subband and the subbands are independent), $\Delta f$ is the subcarrier spacing equal to $\frac{1}{T_{s}}, T_{s}$ denotes the baseband symbol duration and $n(t)$ is the same as $n_{k n}(t)$ above, without the indices. The user index $k$ has been replaced by the subband index $l$, to illustrate the fact that users are not exclusively assigned subbands, but rather contend with each other for the use of each subband according to a multiuser selection diversity algorithm. Moreover, $q$ represents the OFDM symbol and can only take discrete values, while the bit-shaping pulse has been omitted. Intersymbol interference (ISI) is considered to be completely avoided by using the appropriate guard interval. However, the guard interval has not been taken into consideration in computing the total power or the total symbol duration, for the sake of simplicity.

The system model described so far is summarized on Fig. 1, which illustrates a basic SS-MC-MA system. However, subband assignment is not performed in a round-robin (or random) fashion; instead, by employing Channel State Information (CSI), Multi-User Selection Diversity schemes are used (see the next sections).

The channel model properties required to extract the SNIR form have been described above excluding the selfinterference issue. Two models have been devised to study this case, each one leading to a different decision variable.

\section{A. Interference Model I}

Assuming coherent correlation reception, the decision variable for the $j$ th symbol of the $l$ th subband, for the zeroth
OFDM symbol is

$$
R_{l j}=\operatorname{Re}\left\{\sum_{m=1}^{M} w_{l m}(t) c_{l m j} \frac{1}{T_{s}} \int_{0}^{T_{s}} r(t) e^{-j 2 \pi l m \Delta f t} d t\right\}
$$

where $w_{l m}$ are the correlation weights according to selected combining technique.

From (4), after integration the decision variable becomes

$$
R_{l j}=\operatorname{Re}\left\{\sum_{m=1}^{M} w_{m j}(t) r_{m j}\right\} .
$$

Assuming the hypotheses of [16] for single-carrier DSCDMA, this expression for the decision variable is similar to that for MC-DS-CDMA [17]

$$
R_{l j}=d_{l j} \sum_{m=1}^{M} w_{m j} a_{l m} \sqrt{\frac{E_{s}}{M}}+\sum_{m=1}^{M} w_{m j}\left(I_{M_{l m}}+n\right)
$$

where for the purpose of simplicity and without loss of generality, the time representation has been omitted and $I_{M_{l m}}$ represents the interference induced by the remaining $J-1$ user symbols transmitted simultaneously in subband $l$. For a large number of subcarriers and symbols per subband $(M \times(J-1))$, the second term of (6) represents additive white Gaussian noise (AWGN) with zero mean and variance $\sigma_{n}^{2}=N_{0} / 2$ and selfinterference from the symbols of the same user. The latter can be approximated by a Gaussian random variable with zero mean and variance $\sigma_{M_{l m}}^{2}=\frac{(J-1) E_{s} \Omega_{l m}}{2 M}$, where $\Omega_{l m}$ depends on the subband and subcarrier indices, since the users are selected using MUSDiv and matched filtering reception is assumed ${ }^{1}$. Following the analysis in [17], i.e., if AWGN and self-interference are considered independent, an equivalent interference-plus-noise random variable can be defined with power spectral density equal to

$$
\begin{aligned}
\frac{N_{e_{l m}}}{2} & =\sigma_{n}^{2}+\sigma_{M_{l m}}^{2} \\
& =\frac{(J-1) E_{s} \Omega_{l m}}{2 M}+\frac{N_{0}}{2} \\
& =\frac{N_{0}}{2}\left(1+(J-1) \bar{\gamma}_{l m}\right)
\end{aligned}
$$

where $\bar{\gamma}_{l m}=\Omega_{l m}\left(E_{s} / M\right) / N_{0}=\Omega_{l m} E_{b} / N_{0}$ is the average $\mathrm{SNR} /$ symbol of the $l$ th subband and the $m$ th subcarrier. As a result, $R_{l j}$ is a conditionally Gaussian RV with mean

$$
E\left[R_{l j} \mid\left\{a_{l m}\right\}_{m=1}^{M}\right]=\left(\sum_{m=1}^{M} w_{m j} a_{l m}\right) \sqrt{\frac{E_{s}}{M}}
$$

and variance

$$
\operatorname{var}\left(R_{l j} \mid\left\{a_{l m}\right\}_{m=1}^{M}\right)=\sum_{m=1}^{M} w_{m j}^{2} \frac{N_{e_{l m}}}{2} .
$$

Supposing symbols with equal probability, the $l$ th subband conditional SNIR is given by

$$
\operatorname{SNIR}\left(\left\{a_{l m}\right\}_{m=1}^{M}\right)=\frac{\left(E\left[R_{l j} \mid\left\{a_{l m}\right\}_{m=1}^{M}\right]\right)^{2}}{2 \operatorname{var}\left(R_{l j} \mid\left\{a_{l m}\right\}_{m=1}^{M}\right)}
$$

\footnotetext{
${ }^{1}$ This approximation is valid for a large number of interferers and random spreading sequences; the matched filter receiver employed in this work is independent of the signature sequences used, so no actual reference to the type of codes used is made [18].
} 
which, by using (8), (9), results in

$$
\operatorname{SNIR}\left(\left\{a_{l m}\right\}_{m=1}^{M}\right)=\frac{E_{s}}{M} \frac{\left(\sum_{m=1}^{M} w_{m j} a_{l m}\right)^{2}}{\sum_{m=1}^{M} w_{m j}^{2} N_{e_{l m}}} .
$$

The maximum conditional SNIR is achieved by using weighting coefficients according to the Maximal Ratio Combining (MRC) rule

$$
w_{m j}=\frac{E\left[R_{l j} \mid\left\{a_{l m}\right\}_{m=1}^{M}\right]}{\operatorname{var}\left(R_{l j} \mid\left\{a_{l m}\right\}_{m=1}^{M}\right)}=\frac{2 a_{l m}}{N_{e_{l m}}} \sqrt{\frac{E_{s}}{M}} .
$$

Therefore,

$$
\operatorname{SNIR}\left(\left\{a_{l m}\right\}_{m=1}^{M}\right)=\frac{E_{s}}{M} \sum_{m=1}^{M} \frac{a_{l m}^{2}}{N_{e_{l m}}}=\frac{E_{b}}{N_{0}} \sum_{m=1}^{M} \beta_{l m} \gamma_{l m}
$$

with $\beta_{l m}=\frac{1}{1+(J-1) \bar{\gamma}_{l m}}$ and $a_{l m}^{2}=\gamma_{l m}, E_{b}=\frac{E_{s}}{M}$.

Equation (13) can be modified to include the transmitted SNIR

$$
\operatorname{SNIR}\left(\left\{a_{l m}\right\}_{m=1}^{M}\right)=\sum_{m=1}^{M} g_{l m} \gamma_{l m}
$$

where $g_{l m}=\frac{E_{b}}{N_{0}+(J-1) E_{b} \Omega_{l m}}$.

\section{B. Interference Model II}

Under the assumptions made so far, uplink SS-MC-MA is equivalent to downlink MC-CDMA; therefore the analysis in [19] can be followed to approximate the received symbol SNIR. The decision variable in this case follows from (6)

$$
\begin{aligned}
R_{l j}= & d_{l j} \sum_{m=1}^{M} w_{m j} a_{l m} \sqrt{\frac{E_{s}}{M}} \\
& +\sqrt{\frac{E_{s}}{M}} \sum_{m=1}^{M} \sum_{i=1}^{J-1} d_{l i} w_{m j} a_{l m} c_{l m j} c_{l m i} \cos \left(\widehat{\theta}_{m i}\right) \\
& +\eta
\end{aligned}
$$

where $\widehat{\theta}_{m i}=\theta_{m j}-\theta_{m i}$. If MRC and orthogonal codes are used and $\sqrt{\frac{E_{s}}{M}}$ is omitted for simplification purposes, the decision variable becomes

$$
R_{l j}=d_{l j} \sum_{m=1}^{M} a_{l m}^{2}+\sum_{i=1, i \neq j}^{J} d_{l i}\left[\sum_{m=1}^{\frac{M}{2}} a_{l,}^{2}-\sum_{m=1}^{\frac{M}{2}} a_{l, b_{m}}^{2}\right]+\eta
$$

where $a_{m}, b_{m}$ are integer-valued, $a_{m}, b_{m} \in[1, M]$ and $a_{m} \neq$ $b_{m}$.

The second term in (16) is the self-interference which comprises $M \times(J-1)$ interferers. Under Gaussian approximation the variance of the interference term is $\sigma_{i n t}=M(J-1) \Omega_{l}$, where the user has been considered to have the same shortterm average power over all the subcarriers of subband $l$, equal to $\Omega_{l}$.

The third term in (16) represents AWGN which has a total variance of $\sigma_{\eta}=M \Omega_{l}$, where the symbol power has been omitted (considered equal to one). As a result the SNIR per symbol is

$$
\begin{aligned}
\operatorname{SNIR}\left(\left\{a_{l m}\right\}_{m=1}^{M}\right) & =\frac{\left(\sum_{m=1}^{M} a_{l m}^{2}\right)^{2}}{M(J-1) \Omega_{l}^{2}+M \Omega_{l}} \\
& =g_{l}\left(\sum_{m=1}^{M} a_{l m}^{2}\right)^{2}
\end{aligned}
$$

where $g_{l}=\frac{1}{M(J-1) \Omega_{l}^{2}+M \Omega_{l}}$.

If the user average powers in different subcarriers are not identical then the power spectral density is slightly modified as $\sigma_{i n t}=(J-1) \sum_{m=1}^{M} \Omega_{l m}^{2}$.

The noise term is also changed as $\sigma_{\eta}=\sum_{m=1}^{M} \Omega_{l m}$ and the symbol SNIR is given by

$$
\begin{aligned}
\operatorname{SNIR}\left(\left\{a_{l m}\right\}_{m=1}^{M}\right) & =\frac{\left(\sum_{m=1}^{M} a_{l m}^{2}\right)^{2}}{(J-1) \sum_{m=1}^{M} \Omega_{l m}^{2}+\sum_{m=1}^{M} \Omega_{l m}} \\
& =G_{l}\left(\sum_{m=1}^{M} a_{l m}^{2}\right)^{2}
\end{aligned}
$$

where $G_{l}=\frac{1}{(J-1) \sum_{m=1}^{M} \Omega_{l m}^{2}+\sum_{m=1}^{M} \Omega_{l m}}$.

It is quite clear that the main difference between the two models is that, in the first model, the gaussian approximation is performed before applying the MRC rule and interference is extracted for each subcarrier. The opposite is done in the second model where the total interference is extracted (after the combining). As a result, the first model includes the instantaneous gains in both the self-interference and the noise term, while the second model constitutes a more statistical approach. However, the gaussian approximation is performed for $J-1$ terms in the first model and $M(J-1)$ terms in the second one; thus the approximation is more accurate for the latter model.

Hereinafter, Interference Models I and II will be referred to as Models I and II for simplicity.

\section{Capacity}

Expressions (13) and (18) yield the conditional SNIR per symbol. The normalized average conditional system capacity is given by the sum of subband and symbol capacities

$$
C_{\text {total }}=\sum_{l=1}^{L} \sum_{m=1}^{M} \int_{0}^{\infty} \log _{2}\left(1+\gamma_{l}\right) f_{\gamma_{l}}(\gamma) d \gamma
$$

where $\gamma_{l}$ is the subband-symbol conditional SNIR, i.e., $\gamma_{l}$ equals $S N I R$ above. Since all the selected user's symbols are transmitted simultaneously and are subject to identical fading conditions, they all have the same conditional SNIR, thus equation (19) normalized by the number of total symbols yields the normalized average conditional per symbol capacity for the fully loaded case $(J=M)$

$$
C=\frac{1}{M L} \sum_{l=1}^{L} \int_{0}^{\infty} \log _{2}\left(1+\gamma_{l}\right) f_{\gamma_{l}}\left(\gamma_{l}\right) d \gamma_{l} .
$$

From (13) and (18) it is evident that the conditional SNIR is maximized for a given set of user average fading powers when the sum of instantaneous squared channel gains is 


$$
C_{\text {total }}=\frac{J}{(M-1) !} \sum_{l=1}^{L} \sum_{i=1}^{K} \sum_{j=0}^{K-1} \sum_{\substack{i_{1}, i_{2} \ldots i_{j}=1, i_{1}<i_{2}<\ldots<i_{j}, i_{1}, i_{2} \ldots i_{j} \neq i}}^{K} \sum_{\rho_{1}, \rho_{2}, \ldots \rho_{j}=0}^{M-1} \frac{(-1)^{j} D_{1, I}}{\left(g_{i}^{\dagger}\right)^{M}} \int_{0}^{\infty} \log _{2}(1+\zeta) e^{-\zeta\left(\frac{1}{g_{i_{1}}^{\dagger}}+\frac{1}{g_{i_{2}}^{\dagger}}+\ldots+\frac{1}{g_{i_{j}}^{\dagger}}+\frac{1}{g_{i}^{\dagger}}\right)} \zeta^{\rho_{1}+\rho_{2}+\ldots+\rho_{j}+M-1} d \zeta
$$

maximized. Therefore, the average normalized capacity in (20) is maximized when the sum of instantaneous squared channel gains (the sum of instantaneous subchannel SNRs) is maximized for each subband, which is quite intuitive. This concept forms the basis for the algorithms presented in the following sections.

\section{Absolute SNR-BASEd Scheduling}

This algorithm is valid when the user powers are i.i.d. or independent, non-identically distributed (i.non-i.d.) with each other, but each user retains the same average power within one sub-band, i.e. average powers are independent of index $m$.

It is apparent from (14) and (18) that, excluding subchannel average gains (when these gains are independent of the subcarrier index $m$ ), the second case is simplified to the square of the first one. This deduction will be used to extract closedform solutions for the second interference model, based on the first one.

\section{A. Model I}

According to the absolute SNR-based criterion, user $k_{*}$ is selected to transmit all the symbols in subband $l$ for the duration of time slot $t$ if

$$
k_{*}^{l}(t)=\operatorname{argmax}_{k=1 \ldots K}\left\{g_{k} \sum_{m=1}^{M} \gamma_{m l k}\right\} .
$$

The pdf of the instantaneous SNR $\gamma_{m}$ of user $k$ is an exponential distribution ${ }^{2}$ [17]

$$
f_{\gamma_{m k}}(\gamma)=\frac{1}{\Omega_{k}} e^{-\frac{\gamma}{\Omega_{k}}}
$$

and the pdf of the sum of SNRs of user $k$ for subband $l$ is the sum of exponential RVs with the same average power $\Omega_{k}$, if each user is considered to have the same average power for all subbands. If we multiply by the coefficient $g_{k}$ (i.e. $\left.\bar{\gamma}_{k}=g_{k} \sum_{m=1}^{M} \gamma_{m k}\right)$ and define $g_{k}^{\dagger}=g_{k} \Omega_{k}$

$$
f_{\bar{\gamma}_{k_{\max }}}(\gamma)=\frac{\left(\frac{\gamma}{g_{k}^{\dagger}}\right)^{M-1}}{g_{k}^{\dagger}(M-1) !} e^{-\frac{\gamma}{g_{k}^{\dagger}}}, \gamma \geqslant 0
$$

RV $\gamma$, which is commonly used to represent SNR, will be hereinafter denoted by $\zeta$ to avoid confusion with the generalized incomplete gamma function introduced later on.

According to (21), subband $l$ is assigned to the user with the maximum mean (with respect to subcarriers) instantaneous

\footnotetext{
${ }^{2}$ The $l$ th index is omitted as one subband is being studied and each user is assumed to have the same average fading for all subchannels.
}

SNIR. The Cumulative Distribution Function (CDF) of the max of $\bar{\gamma}_{k}$ is given by

$$
F_{\bar{\gamma}_{k_{\max }}}(\zeta)=\prod_{k=1}^{K} F_{\bar{\gamma}_{k}}(\zeta)
$$

where

$$
\begin{aligned}
F_{\bar{\gamma}_{k}}(\zeta) & =\frac{1}{(M-1) !}\left[\Gamma(M)-\Gamma\left(M, \zeta / g_{k}^{\dagger}\right)\right] \\
& =\frac{1}{(M-1) !} \gamma\left(M, \zeta / g_{k}^{\dagger}\right)
\end{aligned}
$$

with $\gamma(a, z)$ being the generalized incomplete gamma function as defined in $[20,6.5 .2]$

The pdf is extracted by differentiating expression (24) with respect to $\zeta$ and after straightforward manipulations

$$
\begin{aligned}
f_{\bar{\gamma}_{k_{\max }}}(\zeta)= & {\left[\frac{1}{(M-1) !}\right]^{K} } \\
& \sum_{i=1}^{K} \frac{\zeta^{M-1}}{\left(g_{i}^{\dagger}\right)^{M}} e^{-\frac{\zeta}{g_{i}^{\dagger}}} \prod_{j=1, j \neq i}^{K} \gamma\left(M, \frac{\zeta}{g_{j}^{\dagger}}\right)
\end{aligned}
$$

Using the finite series representation for the incomplete Gamma function [20, 6.5.11 and 6.5.13] and transforming the product to a sum of products, equation (26) yields

$$
\begin{aligned}
f_{\bar{\gamma}_{k_{\max }}}(\zeta)= & {\left[\frac{1}{(M-1) !}\right]^{K}[(M-1) !]^{K-1} } \\
& \sum_{i=1}^{K} \frac{\zeta^{M-1}}{\left(g_{i}^{\dagger}\right)^{M}} e^{-\zeta / g_{i}^{\dagger}} \sum_{j=0}^{K-1}(-1)^{j} \\
& \times \sum_{\substack{i_{1}, i_{2} \ldots i_{j}=1, i_{1}<i_{2}<\ldots<i_{j}, i_{1}, i_{2} \ldots i_{j} \neq i}}^{K} x_{i_{1}} x_{i_{2}} \cdots x_{i_{j}}
\end{aligned}
$$

where $x_{i_{j}}=e^{-\zeta / g_{i_{j}}^{\dagger}} \sum_{\rho=0}^{M-1} \frac{\left(\zeta / g_{i_{j}}^{\dagger}\right)^{\rho}}{\rho !}$.

By substituting (27) into (19) and exchanging the order of summation and integration, the total capacity is given by (28) at the top of the page. The integral in (28) is not a tabulated one and it is solved in closed form in the Appendix. After manipulations the system capacity becomes

$$
\begin{aligned}
C_{\text {total }}= & \frac{J}{(M-1) ! \ln 2} \sum_{l=1}^{L} \sum_{i=1}^{K} \sum_{j=0}^{K-1} \sum_{\substack{i_{1}, i_{2} \ldots i_{j}=1, i_{1}<i_{2}<\ldots<i_{j}, i_{1}, i_{2} \ldots i_{j} \neq i}}^{K} \\
& \times \sum_{\rho_{1}, \rho_{2}, \ldots \rho_{j}=0}^{M-1} \frac{(-1)^{j} D_{1, I}}{\left(g_{i}^{\dagger}\right)^{M}} A_{1, I}^{-B_{1, I}}
\end{aligned}
$$




$$
\times G_{3,2}^{1,3}\left(\frac{1}{A_{1, I}} \mid \begin{array}{c}
1,1,1-B_{1, I} \\
1,0
\end{array}\right)
$$

where $A_{1, I}=\frac{1}{g_{i_{1}}^{\dagger}}+\frac{1}{g_{i_{2}}^{\dagger}}+\ldots+\frac{1}{g_{i_{j}}^{\dagger}}+\frac{1}{g_{i}^{\dagger}}$,

$B_{1, I}=\rho_{1}+\rho_{2}+\ldots+\rho_{j}+M$,

$D_{1, I}=\frac{1}{\rho_{1} ! \rho_{2} ! \cdots \rho_{j} !} \frac{1}{\left(g_{i_{1}}^{\dagger}\right)^{\rho_{1}}\left(g_{i_{2}}^{\dagger}\right)^{\rho_{2}} \cdots\left(g_{i_{j}}^{\dagger}\right)^{\rho_{j}}}$ and

$G_{p, q}^{m, n}\left(z \mid \begin{array}{l}a_{1}, \ldots a_{n}, a_{n+1}, \ldots, a_{p} \\ b_{1}, \ldots b_{m}, b_{m+1}, \ldots, b_{q}\end{array}\right)$ is the Meijer's $\mathrm{G}$ function [21].

Note, that Meijer's G-function is a standard built-in function in most of the well-known mathematical software packages. In addition, Meijer's G-function can be written in terms of the more familiar generalized hypergeometric functions [21], [22].

\section{B. Model II}

Similar to (21) above, user $k_{*}$ is selected to transmit all the symbols in subband $l$ for the duration of time slot $t$ if

$$
k_{*}^{l}(t)=\operatorname{argmax}_{k=1 \ldots K}\left\{G_{k}\left(\sum_{m=1}^{M} \gamma_{m l k}\right)^{2}\right\}
$$

while the pdf of the sum of SNRs of user $k$ for subband $l$ is

$$
f_{\bar{\gamma}_{k}}(\zeta)=\frac{\left(\frac{\zeta}{\Omega_{k}}\right)^{M-1}}{\Omega_{k}(M-1) !} e^{-\frac{1}{\Omega_{k}} \zeta}, \zeta \geqslant 0 .
$$

The square of $\bar{\gamma}_{k}$ is obtained by using variable $y=\bar{\gamma}_{k}^{2}$ and then multiplying by $G_{k}$ yielding $\bar{\gamma}_{k}=G_{k}\left(\sum_{m=1}^{M} \widehat{\gamma}_{m k}\right)^{2}$. This variable has the pdf defined below

$$
f_{\bar{\gamma}_{k}}(\zeta)=\frac{\left(\frac{\zeta}{G_{k}^{\dagger}}\right)^{\frac{M}{2}-1}}{2 G_{k}^{\dagger}(M-1) !} e^{-\sqrt{\frac{\zeta}{G_{k}^{\dagger}}}}, \zeta \geqslant 0 .
$$

where $G_{k}^{\dagger}=G_{k} \Omega_{k}^{2}$ or $G_{k}^{\dagger}=\frac{\Omega_{k}}{M(J-1) \Omega_{k}+M}$.

Following the same procedure as in Model $I$, the required pdf is

$$
\begin{aligned}
f_{\bar{\gamma}_{k_{\max }}}(\zeta)= & {\left[\frac{1}{(M-1) !}\right]^{K} \sum_{i=1}^{K} \frac{\zeta^{\frac{M}{2}}-1}{2\left(G_{i}^{\dagger}\right)^{\frac{M}{2}}} e^{-\sqrt{\frac{\zeta}{G_{i}^{\dagger}}}} } \\
& \times \prod_{j=1, j \neq i}^{K} \gamma\left(M, \sqrt{\frac{\zeta}{G_{j}^{\dagger}}}\right)
\end{aligned}
$$

and the total capacity is given as

$$
\begin{aligned}
C_{\text {total }}= & \frac{J}{(M-1) !} \sum_{l=1}^{L} \sum_{i=1}^{K} \sum_{j=0}^{K-1} \sum_{\begin{array}{c}
i_{1}, i_{2} \ldots i_{j}=1, \\
i_{1}<i_{2}<\ldots<i_{j}, \\
i_{1}, i_{2} \ldots i_{j} \neq i
\end{array}}^{K} \\
& \sum_{\rho_{1}, \rho_{2, \ldots \rho_{j}}=0}^{M-1} \frac{(-1)^{j} D_{1, I I}}{2\left(G_{i}^{\dagger}\right)^{\frac{M}{2}}} \frac{1}{\sqrt{\pi}} \\
& \times G_{2,4}^{4,1}\left(\frac{\left(A_{1, I I}\right)^{2}}{4} \mid \begin{array}{c}
-\frac{1}{2},-B_{1, I I}, 1-B_{1, I I},-B_{1, I I}
\end{array}\right)
\end{aligned}
$$

where $A_{1, I I}=\frac{1}{\sqrt{G_{i_{1}}^{\prime}}}+\frac{1}{\sqrt{G_{i_{2}}^{\prime}}}+\ldots+\frac{1}{\sqrt{G_{i_{j}}^{\prime}}}+\frac{1}{\sqrt{G_{i}^{\prime}}}$,

$$
\begin{aligned}
& B_{1, I I}=\frac{\rho_{1}+\rho_{2}+\ldots+\rho_{j}+M}{2} \text { and } \\
& D_{1, I I}=\frac{1}{\rho_{1} ! \rho_{2} ! \cdots \rho_{j} ! \cdot\left(G_{i_{1}}^{\dagger}\right)^{\rho_{1} / 2}\left(G_{i_{2}}^{\dagger}\right)^{\rho_{2} / 2} \cdots\left(G_{i_{j}}^{\dagger}\right)^{\rho_{j} / 2}} .
\end{aligned}
$$

\section{NORMALIZED SNR-BASED SCHEDULING}

\section{A. Model I}

According to this criterion, user $k_{*}$ is selected to transmit all the symbols in subband $l$ for the duration of time slot $t$ if

$$
k_{*}^{l}(t)=\operatorname{argmax}_{k=1 \ldots K}\left\{\frac{g_{k}}{\Omega_{k}} \sum_{m=1}^{M} \gamma_{m l k}\right\} .
$$

If the instantaneous SNR is divided by the user average fading power $\Omega_{k}$, the normalized instantaneous SNR $\widehat{\gamma}_{m k}=$ $\gamma_{m k} / \Omega_{k}$ has a pdf independent of $\Omega_{k}$

$$
f_{\widehat{\gamma}_{m k}}(\zeta)=e^{-\zeta}
$$

The pdf of the sum of SNRs of user $k$ for subband $l$ is the sum of exponential random variables with the same average, multiplied by $g_{k}$ (i.e. $\bar{\gamma}_{k}=g_{k} \sum_{m=1}^{M} \widehat{\gamma}_{m k}$ ) to form the criterion (35)

$$
f_{\bar{\gamma}_{k}}(\zeta)=\frac{\left(\zeta / g_{k}\right)^{M-1}}{g_{k}(M-1) !} e^{-\gamma / g_{k}}, \zeta \geqslant 0
$$

which is identical to (23) above except that $g_{k}^{\dagger}$ has been replaced by $g_{k}$. This is the pdf of the sum of normalized SNRs which yields the SNR-related pdf

$$
\begin{aligned}
f_{\bar{\gamma}_{k_{\max }}}\left(\frac{\zeta}{\Omega_{i}}\right)= & {\left[\frac{1}{(M-1) !}\right]^{K} \sum_{i=1}^{K} \frac{\zeta^{M-1}}{\left(\Omega_{i} g_{i}\right)^{M}} e^{-\frac{\zeta}{\Omega_{i} g_{i}}} } \\
& \times \prod_{j=1, j \neq i}^{K} \gamma\left(M, \frac{\zeta}{\Omega_{i} g_{j}}\right)
\end{aligned}
$$

The total capacity is then given by substituting (38) into (19). Therefore, if the analysis in the previous section is followed, the sum capacity yields the same result as in (29) where $A_{1, I}$ and $D_{1, I}$ have been replaced by $A_{2, I}=$ $\frac{1}{\Omega_{i}}\left(\frac{1}{g_{i_{1}}}+\frac{1}{g_{i_{2}}}+\ldots+\frac{1}{g_{i_{j}}}+\frac{1}{g_{i}}\right)$ and $D_{2, I}=\frac{1}{\Omega_{i}^{\rho_{1}+\rho_{2}+\cdots+\rho_{j}} \cdot g_{i_{1}}^{\rho_{1}} g_{i_{2}}^{\rho_{2}} \cdots g_{i_{j}}^{\rho_{j}} \cdot \rho_{1} ! \rho_{2} ! \cdots \rho_{j} !}$ respectively.

\section{B. Model II}

The subband selection criterion is modified by using the Model II $G_{k}$ coefficients and squaring the sum of instantaneous SNRs; user $k_{*}$ is selected to transmit all the symbols in subband $l$ for the duration of time slot $t$

$$
k_{*}^{l}(t)=\operatorname{argmax}_{k=1 \ldots K}\left\{G_{k}\left(\sum_{m=1}^{M} \frac{\gamma_{m l k}}{\Omega_{k}}\right)^{2}\right\} .
$$

Following the above procedure, the pdf of the sum of SNRs of user $k$ for subband $l$ is the sum of exponential random variables with the same average (i.e. $\bar{\gamma}_{k}=\sum_{m=1}^{M} \widehat{\gamma}_{m k}$ ). The square of $\bar{\gamma}_{k}$ is obtained by defining variable $y=\bar{\gamma}_{k}^{2}$ and then 
multiplying by $G_{k}: \bar{\gamma}_{k}=G_{k}\left(\sum_{m=1}^{M} \widehat{\gamma}_{m k}\right)^{2}$. This variable has the pdf below

$$
f_{\bar{\gamma}_{k}}(\zeta)=\frac{\left(\frac{\zeta}{G_{k}}\right)^{\frac{M}{2}-1}}{2 G_{k}(M-1) !} e^{-\sqrt{\frac{\zeta}{G_{k}}}}, \zeta \geqslant 0 .
$$

After manipulations similar to Model $I$, the pdf related to the SNIR is

$$
\begin{aligned}
f_{\bar{\gamma}_{k_{\max }}}\left(\frac{\zeta}{\Omega_{i}^{2}}\right)= & {\left[\frac{1}{(M-1) !}\right]^{K} \sum_{i=1}^{K} \frac{\zeta^{\frac{M}{2}}-1}{2 \Omega_{i}^{M}\left(G_{i}\right)^{\frac{M}{2}}} e^{-\frac{1}{\Omega_{i}} \sqrt{\frac{\zeta}{G_{i}}}} } \\
& \times \prod_{j=1, j \neq i}^{K} \gamma\left(M, \frac{1}{\Omega_{i}} \sqrt{\frac{\zeta}{G_{j}}}\right)
\end{aligned}
$$

The total capacity is given by (34) by replacing $A_{1, I I}, D_{1, I I}$ with

$A_{2, I I}=\frac{1}{\Omega_{i}}\left(\frac{1}{\sqrt{G_{i_{1}}}}+\frac{1}{\sqrt{G_{i_{2}}}}+\ldots+\frac{1}{\sqrt{G_{i_{j}}}}+\frac{1}{\sqrt{G_{i}}}\right)$ and

$D_{2, I I}=\frac{1}{G_{i_{1}}^{\rho_{1} / 2} G_{i_{2}}^{\rho_{2} / 2} \ldots G_{i_{j}}^{\rho_{j} / 2} \cdot \Omega_{i}^{\rho_{1}+\rho_{2}+\ldots+\rho_{j}} \cdot \rho_{1} ! \rho_{2} ! \cdots \rho_{j} !}$ respectively.

\section{SYSTEM NORMALIZED SNR-BASEd SCHEDULING}

\section{A. Model I}

A slightly modified scheme can be produced by changing the normalization procedure of the first algorithm; instead of dividing by the user's average power, the mean system power, excluding the user under consideration (i.e., as if the users were i.i.d.), is used, multiplied by the average user power.

According to this criterion, user $k_{*}$ is selected to transmit all the symbols in subband $l$ for the duration of time slot $t$ if

$$
k_{*}^{l}(t)=\operatorname{argmax}_{k=1 \ldots K}\left\{\frac{g_{k}}{\bar{\Omega}_{k}} \sum_{m=1}^{M} \gamma_{m l k}\right\}
$$

where $\bar{\Omega}_{k}=\sqrt{\frac{\Omega_{k}}{(K-1)} \sum_{i=1, i \neq k}^{K} \Omega_{i}}$

Starting with (23), if $g_{k}^{\dagger}$ is replaced by $g_{k}^{\ddagger}$ to include $\bar{\Omega}_{k}$, i.e. $g_{k}^{\ddagger}=\frac{g_{k} \Omega_{k}}{\bar{\Omega}_{k}}$, then

$$
f_{\bar{\gamma}_{k_{\max }}}(\zeta)=\frac{\left(\frac{\zeta}{g_{k}^{\ddagger}}\right)^{M-1}}{g_{k}^{\ddagger}(M-1) !} e^{-\frac{\zeta}{g_{k}^{\ddagger}}}, \zeta \geqslant 0 .
$$

As a result, the pdf related to the SNR is

$$
\begin{aligned}
f_{\bar{\gamma}_{k_{\max }}}\left(\frac{\zeta}{\bar{\Omega}_{i}}\right)= & {\left[\frac{1}{(M-1) !}\right]^{K} \sum_{i=1}^{K} \frac{\zeta^{M-1}}{\left(\bar{\Omega}_{i} g_{i}^{\ddagger}\right)^{M}} e^{-\frac{\zeta}{\bar{\Omega}_{i} g_{i}^{\ddagger}}} } \\
& \times \prod_{j=1, j \neq i}^{K} \gamma\left(M, \frac{\zeta}{\bar{\Omega}_{i} g_{j}^{\ddagger}}\right)
\end{aligned}
$$

Therefore, if the analysis in the previous section is followed, the sum capacity yields the same result as (29) by substituting $A_{1, I}$ and $D_{1, I}$ with $A_{3, I}=\frac{1}{\bar{\Omega}_{i}}\left(\frac{1}{g_{i_{1}}^{\ddagger}}+\frac{1}{g_{i_{2}}^{\ddagger}}+\ldots+\frac{1}{g_{i_{j}}^{\ddagger}}+\frac{1}{g_{i}^{\ddagger}}\right)$ and

$D_{3, I}=\frac{1}{\rho_{1} ! \rho_{2} ! \cdots \rho_{j} !} \frac{1}{\left(g_{i_{1}}^{\ddagger}\right)^{\rho_{1}}\left(g_{i_{2}}^{\ddagger}\right)^{\rho_{2}} \ldots\left(g_{i_{j}}^{\ddagger}\right)^{\rho_{j}}} \frac{1}{\bar{\Omega}_{i}^{\rho_{1}+\rho_{2}+\ldots+\rho_{j}}}$ respectively.

\section{B. Model II}

The criterion is satisfied if user $k_{*}$ is selected to transmit all the symbols in subband $l$ for the duration of time slot $t$

$$
k_{*}^{l}(t)=\operatorname{argmax}_{k=1 \ldots K}\left\{G_{k}\left(\sum_{m=1}^{M} \frac{\gamma_{m l k}}{\bar{\Omega}_{k}}\right)^{2}\right\} .
$$

Following a similar procedure for the second model the pdf becomes

$$
\begin{aligned}
f_{\bar{\gamma}_{k_{\max }}}\left(\frac{\zeta}{\bar{\Omega}_{i}^{2}}\right)= & {\left[\frac{1}{(M-1) !}\right]^{K} \sum_{i=1}^{K} \frac{\zeta^{\frac{M}{2}}-1}{2 \bar{\Omega}_{i}^{M}\left(G_{i}^{\ddagger}\right)^{\frac{M}{2}}} e^{-\frac{1}{\bar{\Omega}_{i}}} \sqrt{\frac{\zeta}{G_{i}^{\ddagger}}} } \\
& \times \prod_{j=1, j \neq i}^{K} \gamma\left(M, \frac{1}{\bar{\Omega}_{i}} \sqrt{\frac{\zeta}{G_{j}^{\ddagger}}}\right) .
\end{aligned}
$$

and the capacity is given by (34) by replacing $A_{1, I I}, D_{1, I I}$

$A_{3, I I}=\frac{1}{\bar{\Omega}_{i}}\left(\frac{1}{\sqrt{G_{i_{1}}^{\ddagger}}}+\frac{1}{\sqrt{G_{i_{2}}^{\ddagger}}}+\ldots+\frac{1}{\sqrt{G_{i_{j}}^{\ddagger}}}+\frac{1}{\sqrt{G_{i}^{\ddagger}}}\right)$ and

$D_{3, I I}=$

$\frac{1}{\left(G_{i_{1}}^{\ddagger}\right)^{\rho_{1} / 2}\left(G_{i_{2}}^{\ddagger}\right)^{\rho_{2} / 2} \cdots\left(G_{i_{j}}^{\ddagger}\right)^{\rho_{j} / 2} \cdot \bar{\Omega}_{i}^{\rho_{1}+\rho_{2}+\ldots+\rho_{j}} \cdot \rho_{1} ! \rho_{2} ! \cdots \rho_{j} !}$ respectively.

\section{AbSOlute SNR-BASED SCHEDULING FOR Non-IdENTICAL SUbCARRIER POWER PROFILES}

\section{A. Model I}

In this scenario each user is considered to have distinct average fading power coefficients for each subcarrier $m$ and each subband $l$. According to the absolute SNR-based criterion, user $k_{*}$ is selected to transmit all the symbols in subband $l$ for the duration of time slot $t$ if

$$
k_{*}^{l}(t)=\operatorname{argmax}_{k=1 \ldots K}\left\{\sum_{m=0}^{M-1} \frac{\gamma_{m l k}}{N_{m l k}}\right\}
$$

where the average SNIR $N_{m l k}=\frac{N_{0}}{E_{b}}+(J-1) \Omega_{m l k}$ has been inserted inside the summation since it is dependent on $m$.

The pdf of the instantaneous SNR $\gamma_{m l}$ of user $k$ is an exponential distribution, but each one has a different average fading power $\Omega_{m l k}$ for every subchannel (defined by subband $(l)$ and subcarrier $(m)$ coefficients), so the pdf of the SNIR/subcarrier $\left(\widehat{\gamma}_{m l k}=\frac{\gamma_{m l k}}{N_{m l k}}\right)$ has a coefficient $\frac{1}{g_{m l k}}$ with $g_{m l k}=\frac{\Omega_{m l k}}{N_{m l k}}=\frac{E_{b} \Omega_{m l k}}{N_{0}+(J-1) E_{b} \Omega_{m l k}}$.

The pdf of the sum of SNRs of user $k$ for subband $l$ is the sum of exponential random variables with different average fading powers $g_{m l k} \bar{\gamma}_{l k}=\sum_{m=1}^{M} \widehat{\gamma}_{m l k}$ which yields a hypoexponential distribution [23]

$$
f_{\bar{\gamma}_{l k}}(\zeta)=\sum_{m=1}^{M} \frac{1}{g_{m l k}} b_{m l k} e^{-\frac{1}{g_{m l k}} \zeta}, \zeta \geqslant 0
$$

where $b_{m l k}=\prod_{j=1, j \neq m}^{M} \frac{g_{m l k}}{g_{m l k}-g_{j l k}}$. 


$$
C_{\text {total }}=\frac{J}{\ln 2} \sum_{l=1}^{L} \sum_{m_{1}, m_{2}, \ldots, m_{K}=1}^{M} B_{4, I I} \cdot \sum_{r=1}^{K} \frac{2}{\sqrt{G_{m_{r} l r}}} \sum_{p=0}^{K-1}(-1)^{p} \sum_{\substack{n_{1}, n_{2} \ldots n_{p}=1, n_{1}<n_{2}<\ldots<n_{p}, n_{1}, n_{2} \ldots n_{p} \neq r}}^{K} \frac{\sin A_{4, I I}\left(\frac{\pi}{2}-\operatorname{Si}\left(A_{4, I I}\right)\right)-\cos A_{4, I I} \operatorname{Ci}\left(A_{4, I I}\right)}{A_{4, I I}}
$$

The CDF of the max of $\bar{\gamma}_{l k}$ is $F_{\bar{\gamma}_{k_{\max }}}(\zeta)=\prod_{k=1}^{K} F_{\bar{\gamma}_{l k}}(\zeta)$, where $F_{\bar{\gamma}_{l k}}(\gamma)=\sum_{m=1}^{M} b_{m l k}\left(1-e^{-\frac{1}{g_{m l k}} \zeta}\right)$, so the pdf is produced by differentiating $F_{\bar{\gamma}_{k_{\max }}}(\zeta)$ with respect to $\zeta$

$$
\begin{aligned}
f_{\bar{\gamma}_{k_{\max }}}(\zeta)= & \sum_{m_{1}, m_{2}, \ldots, m_{K}=1}^{M} b_{m_{1} l 1} b_{m_{2} l 2} \cdots b_{m_{K} l K} \\
& \sum_{r=1}^{K} \frac{1}{g_{m_{r} l r}} e^{-\frac{1}{g_{m_{r} l r}} \zeta} \\
& \times \prod_{p=1, p \neq r}^{K}\left(1-e^{-\frac{1}{g_{m_{p} l p}} \zeta}\right) .
\end{aligned}
$$

By employing the previous manipulations, the average sum capacity becomes

$$
\begin{aligned}
C_{\text {total }}= & \frac{J}{\ln 2} \sum_{l=1}^{L} \sum_{m_{1}, m_{2}, \ldots, m_{K}=1}^{M} B_{4, I} \sum_{r=1}^{K} \frac{1}{g_{m_{r} l r}} \sum_{p=0}^{K-1}(-1)^{p} \\
& \sum_{\substack{n_{1}, n_{2} \ldots n_{p}=1, n_{1}<n_{2}<\ldots<n_{p}, n_{1}, n_{2} \ldots n_{p} \neq r}}^{K} \frac{e^{A_{4, I}} E_{1}\left(A_{4, I}\right)}{A_{4, I}}
\end{aligned}
$$

where $A_{4, I}=\frac{1}{g_{m_{r} l r}}+\frac{1}{g_{m_{n_{1}} l_{1}}}+\frac{1}{g_{m_{n} n_{2} l_{2}}}+\ldots+\frac{1}{g_{m_{n_{p}} n_{p}}}$, $B_{4, I}=b_{m_{1} l 1} b_{m_{2} l 2} \cdots b_{m_{K} l K}$ and $E_{1}(x)$ is the exponential integral.

\section{B. Model II}

With the usual modification for Model II, user $k_{*}$ is selected to transmit all the symbols in subband $l$ for the duration of time slot $t$ if

$$
k_{*}^{l}(t)=\operatorname{argmax}_{k=1 \ldots K}\left\{G_{k}\left(\sum_{m=0}^{M-1} \gamma_{m l k}\right)^{2}\right\} .
$$

The pdf of the sum of SNRs is [23]

$$
f_{\bar{\gamma}_{l k}}(\zeta)=\sum_{m=1}^{M} \frac{1}{\Omega_{m l k}} b_{m l k} e^{-\frac{1}{\Omega_{m l k}} \zeta}, \zeta \geqslant 0
$$

where $b_{m l k}=\prod_{j=1, j \neq m}^{M} \frac{\Omega_{m l k}}{\Omega_{m l k}-\Omega_{j l k}}$.

The square of $\bar{\gamma}_{l k}$ is obtained by variable $y=\bar{\gamma}_{l k}^{2}$ and then multiplying by $G_{k}$, yielding $\bar{\gamma}_{k}=G_{k}\left(\sum_{m=1}^{M} \widehat{\gamma}_{m k}\right)^{2}$, where $G_{k}=\frac{1}{(J-1) \sum_{m=1}^{M} \Omega_{m l k}^{2}+\sum_{m=1}^{M} \Omega_{m l k}}$.
This variable has a pdf defined below

$$
f_{\bar{\gamma}_{k}}(\zeta)=\frac{1}{2 \sqrt{\zeta}} \sum_{m=1}^{M} \frac{1}{\sqrt{G_{m l k}}} b_{m l k} e^{-\sqrt{\frac{\zeta}{G_{m l k}}}}, \zeta \geqslant 0
$$

if $G_{m l k}=G_{k} \Omega_{m l k}^{2}$.

The pdf of the SNIR results in

$$
\begin{aligned}
f_{\bar{\gamma}_{k_{\max }}}(\zeta)= & \sum_{m_{1}, m_{2}, \ldots, m_{K}=1}^{M} b_{m_{1} l 1} b_{m_{2} l 2} \cdots b_{m_{K} l K} \\
& \sum_{r=1}^{K} \frac{1}{2 \sqrt{\zeta}} \frac{1}{\sqrt{G_{m_{r} l r}}} e^{-\sqrt{\frac{\zeta}{G_{m_{r} l r}}}} \\
& \times \prod_{p=1, p \neq r}^{K}\left(1-e^{-\sqrt{\frac{\zeta}{G_{m_{p} l p}}}}\right)
\end{aligned}
$$

which leads to the average sum capacity (55) at the top of the page (the integral is a simplified form of the one solved in the Appendix), where $B_{4, I I}=b_{m_{1} l 1} b_{m_{2} l 2} \cdots b_{m_{K} l K}$, $A_{4, I I}=\frac{1}{\sqrt{G_{m_{r} l r}}}+\frac{1}{\sqrt{G_{m_{n}} l_{1}}}+\frac{1}{\sqrt{G_{m_{n_{2}} l n_{2}}}}+\ldots+\frac{1}{\sqrt{G_{m_{n}} l_{n_{p}}}}$ and $\mathrm{Si}()$ and $\mathrm{Ci}()$ are the sine and cosine integrals respectively.

\section{NORMALIZED SCHEMES FOR NON-IDENTICAL SubCARrier POWER Profiles}

\section{A. Model I}

Modifications have to be made for the application of the non-identical subcarrier profile scenario to the two normalized schemes. Let us define the user subband average power

$$
\underline{\Omega}_{k}=\frac{1}{M} \sum_{m=1}^{M} \Omega_{m}
$$

The normalized SNR-based scheduling algorithm states that user $k_{*}$ is selected to transmit all the symbols in subband $l$ for the duration of time slot $t$ if

$$
k_{*}^{l}(t)=\operatorname{argmax}_{k=1 \ldots K}\left\{\frac{1}{\underline{\Omega}_{k}} \sum_{m=0}^{M-1} \frac{\gamma_{m l k}}{N_{m l k}}\right\}
$$

yielding the capacity expression in (50), where $A_{4, I}$ is replaced by

$A_{5, I}=\frac{1}{\underline{\Omega}_{r}}\left(\frac{1}{g_{m_{r} l r}}+\frac{1}{g_{m_{n_{1}} l n_{1}}}+\frac{1}{g_{m_{n_{2}} l_{2}}}+\ldots+\frac{1}{g_{m_{n} n_{p} l n_{p}}}\right)$.

In the same fashion, the system-normalized SNR-based Algorithm rules that user $k_{*}$ is selected to transmit all the symbols in subband $l$ for the duration of time slot $t$

$$
k_{*}^{l}(t)=\operatorname{argmax}_{k=1 \ldots K}\left\{\frac{1}{\overline{\underline{\Omega}}_{k}} \sum_{m=0}^{M-1} \frac{\gamma_{m l k}}{N_{m l k}}\right\}
$$




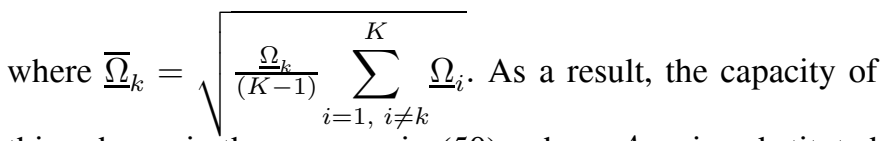
this scheme is the same as in (50), where $A_{4, I}$ is substituted by $A_{6, I}=\frac{1}{\underline{\bar{s}}_{r}}\left(\frac{1}{g_{m_{r} l r}}+\frac{1}{g_{m_{n_{1}} l_{1}}}+\frac{1}{g_{m_{n_{2}} l_{2}}}+\ldots+\frac{1}{g_{m_{n_{p}} l_{p}}}\right)$.

\section{B. Model II}

The same notion is applied to the second model by defining the user subband average squared power

$$
\widehat{\Omega}_{k}=\frac{1}{M} \sum_{m=1}^{M} \Omega_{m}^{2}
$$

so that the normalized scheme becomes

$$
k_{*}^{l}(t)=\operatorname{argmax}_{k=1 \ldots K}\left\{\frac{G_{l}}{\widehat{\Omega}_{k}}\left(\sum_{m=0}^{M-1} \gamma_{m l k}\right)^{2}\right\}
$$

which produces the same capacity expression as (55) by substituting $A_{4, I I}$ with

$$
\begin{aligned}
A_{5, I I}= & \frac{1}{\sqrt{\widehat{\Omega}_{r}}}\left(\frac{1}{\sqrt{G_{m_{r} l r}}}+\frac{1}{\sqrt{G_{m_{n_{1}} l n_{1}}}}+\frac{1}{\sqrt{G_{m_{n_{2}} l n_{2}}}}+\ldots\right. \\
& \left.+\frac{1}{\sqrt{G_{m_{n_{p}} l n_{p}}}}\right)
\end{aligned}
$$$$
\text { If } \underline{\widehat{\Omega}}_{k}=\frac{\widehat{\Omega}_{k}}{(K-1)} \sum_{i=1, i \neq k}^{K} \widehat{\Omega}_{i} \text {, the system-normalized scheme }
$$
chooses user $k$ such that

$$
k_{*}^{l}(t)=\operatorname{argmax}_{k=1 \ldots K}\left\{\frac{G_{k}}{\widehat{\widehat{\Omega}}_{k}}\left(\sum_{m=0}^{M-1} \gamma_{m l k}\right)^{2}\right\}
$$

leading to (55) for

$$
\begin{aligned}
& A_{6, I I}=\frac{1}{\sqrt{\widehat{\Omega}_{r}}} \\
& \times\left(\frac{1}{\sqrt{G_{m_{r} l r}}}+\frac{1}{\sqrt{G_{m_{n_{1}} l n_{1}}}}+\frac{1}{\sqrt{G_{m_{n_{2}} l_{2}}}}+\ldots+\frac{1}{\sqrt{G_{m_{n_{p}} n_{p}}}}\right) \\
& \text { replacing } A_{4, I I} .
\end{aligned}
$$

\section{Numerical RESUlts AND Discussion}

Computer simulations were performed to ensure the validity of the presented mathematical analysis. The transmitted SNR per baseband symbol is set to unity for simplicity. The algorithms are tested for a number of users ranging from 2 to 16 , assuming systems with $L=M=8$ and $L=M=16$. Both systems are considered fully loaded $(J=M)$. The users' power profiles are logarithmic, ranging from 0 to $10 \mathrm{~dB}$ for the i.non-i.d case, but normalized so that the average user power (if they were i.i.d) is $5 \mathrm{~dB}$ regardless of the number of users, in order to conduct a fair comparison. In the general case (where each user is considered to have distinct average fading power coefficients for each subcarrier $m$ and each subband $l$ ) the power profile again falls into the aforementioned pattern. A simulation of the SS-MC-MA system without any scheduling scheme is also included for comparison. The random selection and the absolute scheme were tested for both i.i.d and i.noni.d user profiles; It is apparent that the normalized schemes

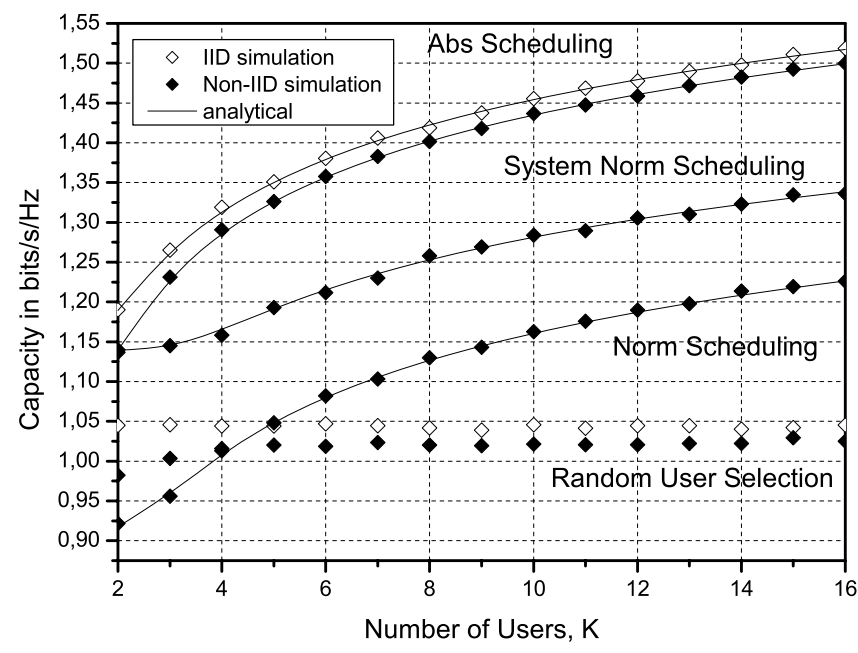

Fig. 2. Average Normalized per Symbol Capacity for $L=M=8$, Model I.

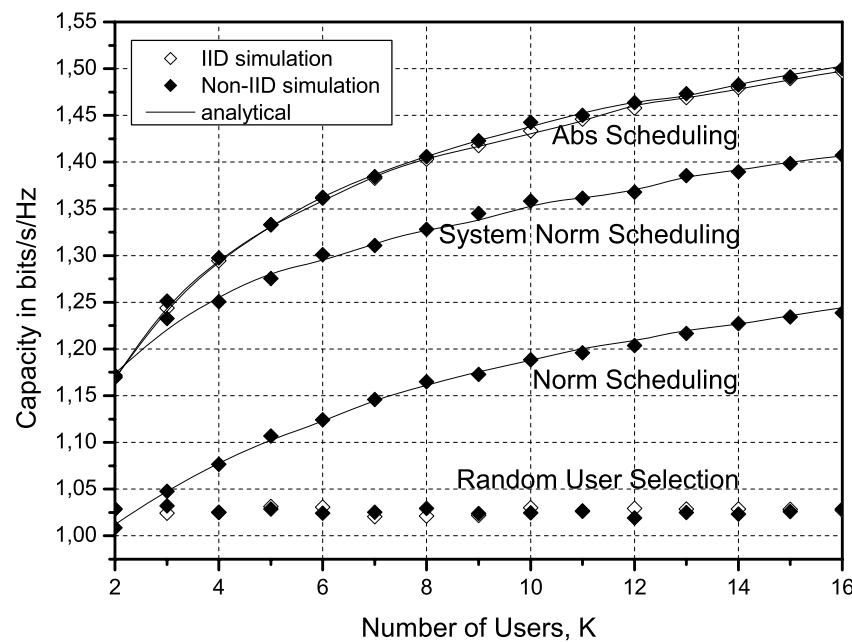

Fig. 3. Average Normalized per Symbol Capacity for $L=M=8$, Model I, i.non-i.d subcarrier power profile.

attain the same results for both i.i.d. and i.non-i.d cases. The analytical results are depicted on the same plots.

All the plots fulfill the intuitive expectation that the absolute SNR-based scheduling achieves the highest average normalized "per symbol" capacity among the tested algorithms, with the system-normalized SNR-based scheduling scheme outperforming the normalized scheme. The latter scheme increases capacity compared to the standard normalized scheme, but also maintains a certain degree of fairness compared the absolute scheme; the normalization coefficient is balanced by including both the average power of the specific user and the powers of the rest of the users. Note that for $K=2$ the systemnormalized scheme does not constitute a separate case; it is exactly the same as the absolute algorithm. Therefore, any comments referring to it are valid for $K>2$. Theory and simulation results agree on all accounts.

The first four plots refer to the $L=M=8$ system, so the number of users can be greater than the number of available subbands. It is obvious that, as the number of users increases up to nearly the number of available subbands, 


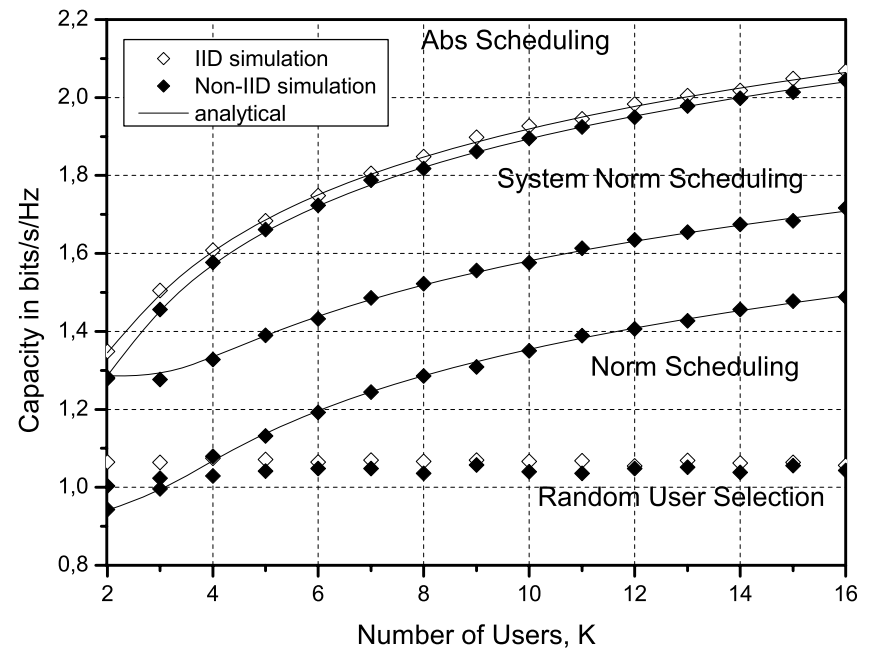

Fig. 4. Average Normalized per Symbol Capacity for $L=M=8$, Model II.

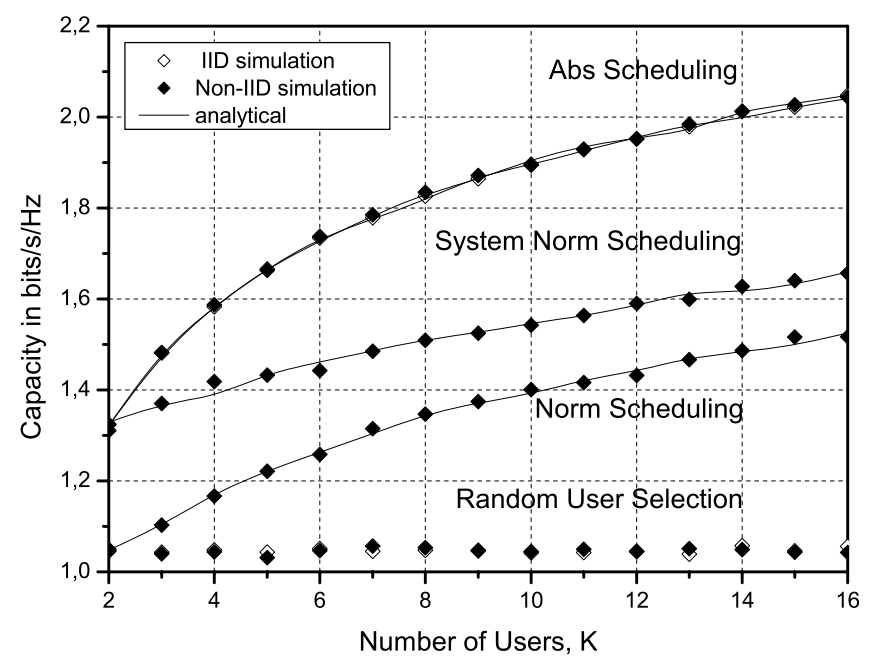

Fig. 5. Average Normalized per Symbol Capacity for $L=M=8$, Model II, i.non-i.d subcarrier power profile

the absolute and normalized scheme capacity show a sharp increase, an effect which dwindles as the number of users passes that threshold. The system-normalized scheme which is equivalent to the absolute scheme for $K=2$, shows the same behavior for non-identical subcarrier profile and the opposite one for identical subcarrier profile. The extra randomness induced in the non-identical case appears to account for this behavior. This randomness also appears to alleviate the difference between the i.i.d user power profile and the i.non-i.d one for the two involved schemes. For a low number of users the normalized scheme can even reduce the system capacity compared to random user selection. Finally, the second interference model, depicted in Fig. 4-5, is clearly more optimistic due to the fact that interference is studied statistically, while the signal is extracted from actual instantaneous values. As a result, when the best user is selected, the best signal is selected and interference remains the same, while, in reality, it should increase as well. Another difference between the two models is that the system-normalized algorithm plots are closer to the absolute algorithm in the first model as opposed to the second

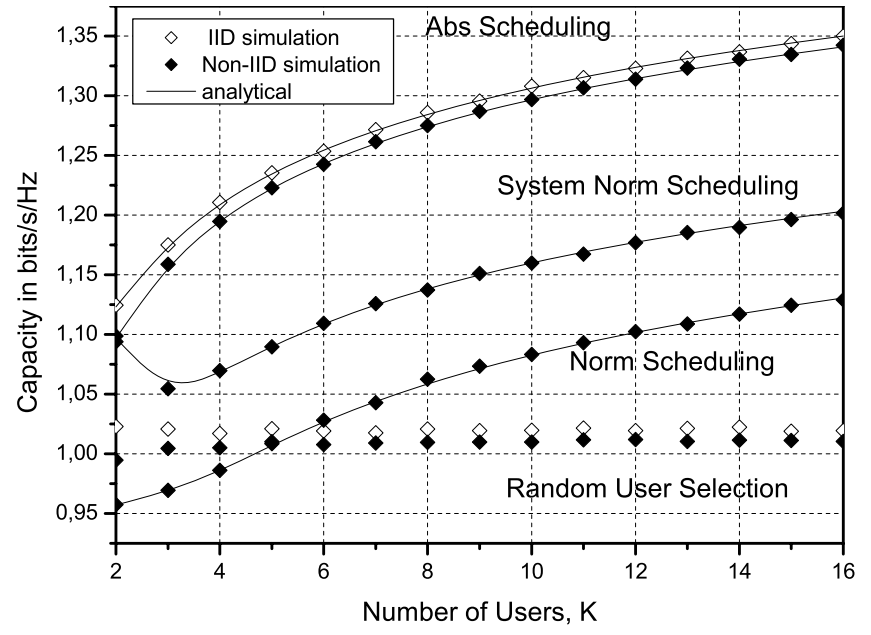

Fig. 6. Average Normalized per Symbol Capacity for $L=M=16$, Model

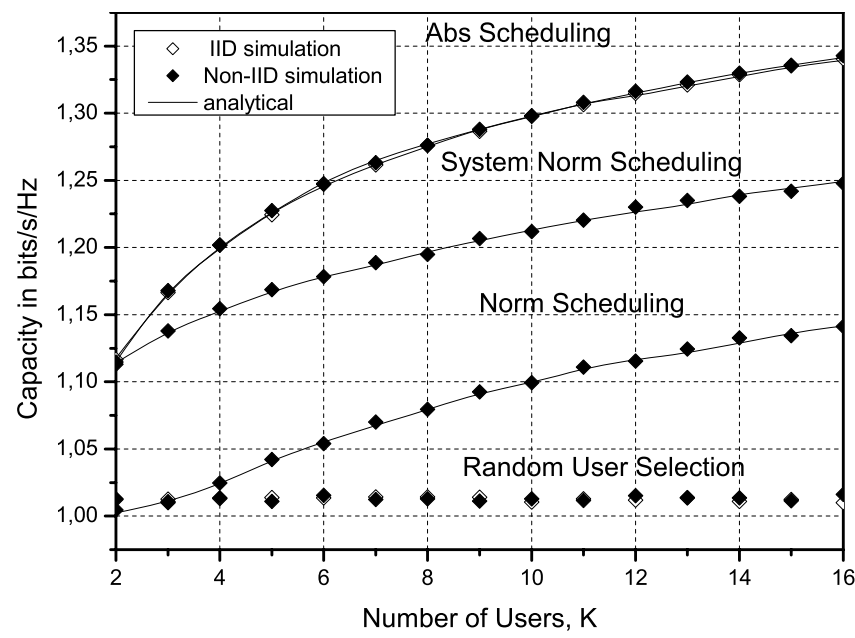

Fig. 7. Average Normalized per Symbol Capacity for $L=M=16$, Model I, i.non-i.d subcarrier power profile.

one. This can be ascribed to the fact that the normalization coefficient is squared in the second model, thus being more effective regarding fairness.

The last four figures examine the $L=M=16$ system. This system achieves a lower overall performance, compared to the previous case for the same range of users. However, it produces similar results if the comparison is carried out, bearing in mind the no. of users/no. of subbands ratio, e.g. all the schemes for the first system for $K=2-8$ obtain close, yet slightly superior, results to the ones in the second system for $K$ ranging from 2 to 16 . This second set of figures is generally more pessimistic, but also more accurate; the system parameters are all doubled, a fact which further justifies the gaussian approximations in both models.

\section{CONCLUSION}

The application of two known and one novel multi-user selection diversity algorithms was examined for the SS-MCMA system. All three algorithms were tested for both i.i.d. and i.non-i.d user power distributions, for both identical and non-identical subcarrier power distributions per user, for 


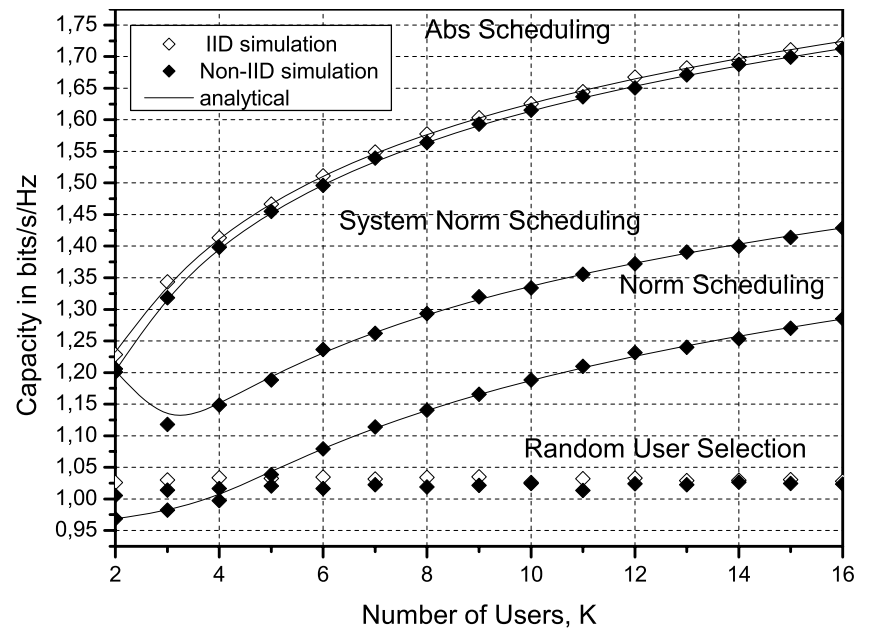

Fig. 8. Average Normalized per Symbol Capacity for $L=M=16$, Model II.

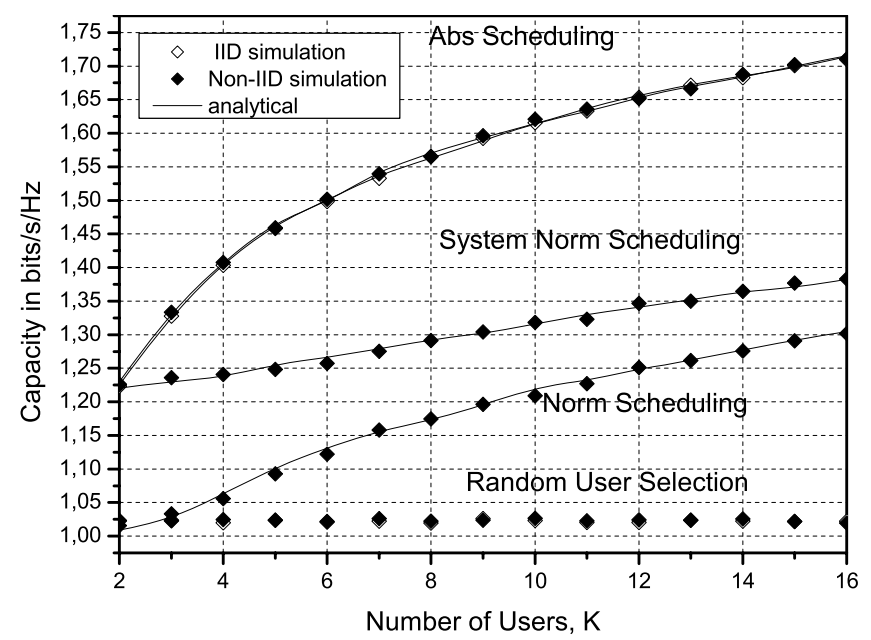

Fig. 9. Average Normalized per Symbol Capacity for $L=M=16$, Model II, i.non-i.d subcarrier power profile.

two interference approximation models, under two SS-MCMA system architectures. Closed-form expressions for the system capacity were derived for all the presented schemes, which were corroborated by ample numerical simulations. The Gaussian approximation in the interference calculation is reasonable under the selected system design, a fact which is validated by the close results obtained from two different systems. Although the algorithms are optimized depending on the selected interference model, the basic concept is that each scheme opts for the user that maximizes the absolute/normalized sum of the subcarrier powers. The algorithms increase average normalized per symbol capacity compared to random user selection, each one offering a different trade-off between capacity and fairness. Another obvious advantage is clearly obtained from the fact that channel state information is mandatory for MC-CDMA-like systems, so the extra burden of overhead data required for effective MUSDiv is greatly reduced. As an overall conclusion, it is evident that MUSDiv can be beneficial to frequency-diversity-related CDMA systems as well as to TDMA ones.

\section{APPENDIX \\ INTEGRAL EVALUATION}

The following integral

$$
I_{1}=\int_{0}^{\infty} x^{z-1} e^{-a x} \log _{2}(1+x) d x
$$

can be evaluated in closed-form by using the Meijer's G representations

$$
\begin{aligned}
\log _{2}(1+x) & =\frac{1}{\ln 2} G_{2,2}^{1,2}\left(x \mid \begin{array}{l}
1,1 \\
1,0
\end{array}\right) \\
e^{-a x} & =G_{0,1}^{1,0}(a x \mid 0) .
\end{aligned}
$$

Thus [21]

$$
\begin{aligned}
I_{1} & =\frac{1}{\ln 2} \int_{0}^{\infty} G_{0,1}^{1,0}(a x \mid 0) \cdot G_{2,2}^{1,2}\left(x \mid \begin{array}{c}
1,1 \\
1,0
\end{array}\right) x^{z-1} d x \\
& =\frac{1}{\ln 2} a^{-z} G_{3,2}^{1,3}\left(\frac{1}{a} \mid \begin{array}{c}
1,1,1-z \\
1,0
\end{array}\right)
\end{aligned}
$$

If the integral is of the form

$$
I_{2}=\int_{0}^{\infty} x^{z-1} e^{-a \sqrt{x}} \log _{2}(1+x) d x
$$

then by following the same procedure as above

$$
I_{2}=\frac{1}{\ln 2} \frac{1}{\sqrt{\pi}} G_{2,4}^{4,1}\left(\frac{a^{2}}{4} \mid \begin{array}{c}
-z, 1-z \\
0, \frac{1}{2},-z,-z
\end{array}\right) .
$$

\section{ACKNOWLEDGEMENT}

The authors wish to thank the Editor and the anonymous reviewers for their valuable comments.

\section{REFERENCES}

[1] R. Knopp and P. Humblet, "Information capacity and power control in single-cell multiuser communications," in Proc. IEEE Int. Commun. Conf. (ICC'95), pp. 331-335, June 1995.

[2] D. N. C. Tse, "Optimal power allocation over parallel Gaussian channels," in Proc. Int. Symp. Inform. Theory (ISIT'97), p. 27, June 1997, Ulm, Germany.

[3] P. Viswanath, D. Tse, and R. Laroia, "Opportunistic beamforming using dumb antennas," IEEE Trans. Inform. Theory, vol. 48, pp. 1277-1294, June 2002.

[4] D. Piazza and L. Milstein, "Multiuser diversity-mobility tradeoff: modeling and performance analysis of a proportional fair scheduling," in Proc. IEEE Global Telecommun. (Globecom '02), vol. 1, pp. 906-910, Nov. 2002, Taipei, Taiwan.

[5] L. Yang and M.-S. Alouini, "Performance analysis of multiuser selection diversity," in Proc. IEEE Int. Conf. Commun. (ICC'04), pp. 3066-3070, June 2004, Paris, France.

[6] D. Gesbert and M. S. Alouini, "How much feedback is multi-user diversity really worth," in Proc. IEEE Int. Conf. Commun. (ICC'04), June 2004, Paris, France.

[7] B. Holter, M.-S. Alouini, G. E. Øien, and H.-C. Yang, "Multiuser switched diversity transmission," in Proc. Vehicular Technology Conference (VTC2004), vol. 3, pp. 2038-2043, Sept. 2004, Los Angeles, CA.

[8] L. Yang, M.-S. Alouini, and D. Gesbert, "Further results on selective multiuser diversity," in Proc. 7th ACM/IEEE International Symposium on Modeling, Analysis and Simulation of Wireless and Mobile Systems (MSWiM 2004), pp. 25-30, Oct. 2004, Venezia, Italy.

[9] C. Y. Wong, R. S.Cheng, K. B. Letaief, and R. D. Murch, "Multiuser OFDM with adaptive subcarrier, bit, and power allocation," IEEE $J$. Select. Areas Commun., vol. 17, no. 10, pp. 1747-1758, Oct. 1999.

[10] L. Xiao, S. Zhou, and Y. Yao, "QoS-oriented scheduling algorithm for mobile multimedia In OFDM," in Proc. PIMRC 2003, vol. 1, pp. 545549, Sept. 2003, Beijing, China. 
[11] L. Xiao, A. Wang, S. Zhou, and Y. Yao, "A dynamic resource scheduling algorithm for OFDM system," in Proc. 9th Asia-Pacific Communication Conference, vol. 2, pp. 444-447, Sept. 2003, Malaysia.

[12] R. van Nee and R. Prasad, On Limits of Wireless Communications in a Fading Environment when Using Multiple Antennas. New York: Artech House, 2000.

[13] S. Kaiser and K. Fazel, "A flexible spread-spectrum multicarrier multiple-access system for multi-media communications," in Proc. IEEE PIMRC '97, pp. 100-104, Sept. 1997, Helsinki, Finland.

[14] J. G. Proakis, Digital Communications, 3rd ed. New York: McGrawHill, 1995.

[15] Z. Hou and V. Dubey, "Exact analysis for downlink MC-CDMA in Rayleigh fading channels," IEEE Commun. Lett., vol. 8, no. 2, pp. 90 92, Feb. 2004.

[16] S. Kondo and L. B. Milstein, "Performance of multicarrier DS-CDMA systems," IEEE Trans. Commun., vol. 44, no. 2, pp. 238-246, Feb. 1996.

[17] M. K. Simon and M.-S. Alouini, Digital Communication over Fading Channels, 2nd ed. Wiley-Interscience, 2005.

[18] D. N. C. Tse, "Linear multiuser receivers: effective interference, effective bandwidth and user capacity ," IEEE Trans. Inform. Theory, vol. 45, no. 2, pp. 641-657, Mar. 1999.

[19] N. Yee and J.-P. Linnartz, "Multi-carrier CDMA in indoor wireless radio networks," in Proc. PIMRC '93, pp. 109-113, 1993, Yokohama, Japan.

[20] M. Abramowitz and I. A. Stegun, Handbook of Mathematical Functions with Formulas, Graphs and Mathematical Tables, 9th ed. New York: Dover Publications, 1970.

[21] V. Adamchik and O. I. Marichev, "The algorithm for calculating integrals of hypergeometric type functions and its realization in reduce system," in Proc. International Symposium on Symbolic and Algebraic Computation, vol. 1, pp. 212-224, 1990, Tokyo, Japan.

[22] I. S. Gradshteyn and I. M. Ryzhik, Table of Integrals, Series, and Products, 6th ed. New York: Academic, 2000.

[23] S. Ross, Introduction to Probability Models, 6th ed. San Diego: Academic Press, 1997.

[24] J. Jang and K. B. Lee, "Transmit power adaptation for multiuser OFDM systems," IEEE J. Select. Areas Commun., vol. 21, no. 2, pp. 171-178, Feb. 2003.

[25] W. C. Y. Lee, "Estimate of channel capacity in Rayleigh fading environment," IEEE Trans. Veh. Technol., vol. 39, no. 3, pp. 187-190, Aug. 1990.

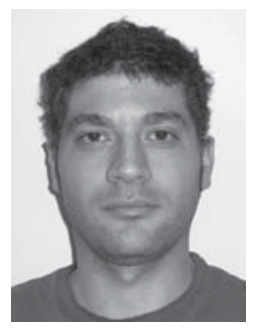

Petros L. Katsis received his Diploma in Electrical and Computer Engineering from the Aristotle University of Thessaloniki, Greece in 2002. He is currently a $\mathrm{PhD}$ candidate in the same department where he also serves as a teaching assistant. His research interests include multicarrier systems in multipath, fading channels such as wireless and powerline communications. Mr Katsis participates in the IST Integrated Project OPERA, in the NoE SATNEX, and in COST Action 289. He is a member of the IEEE and a member of the Technical Chamber

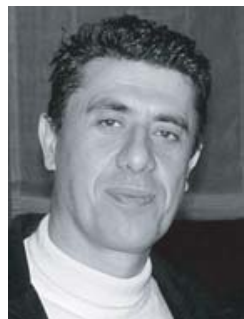

George K. Karagiannidis (M'97-SM'04) was born in Pithagorion, Samos Island. He received the University and Ph.D. degrees in electrical engineering from the University of Patras, Patras, in 1987 and 1999, respectively.

From 2000 to 2004, he was a Senior Researcher at the Institute for Space Applications and Remote Sensing, National Observatory of Athens, Greece. In June 2004, he joined Aristotle University of Thessaloniki, Thessaloniki, where he is currently an Assistant Professor in the Electrical and Computer

Engineering Department.

His current research interests include wireless communication theory, digital communications over fading channels, cooperative diversity systems, cognitive radio, satellite communications, and wireless optical communications. He is the author or coauthor of more than 80 technical papers published in scientific journals and presented at international conferences. He is also a coauthor of two chapters in books and a coauthor of the Greek edition of a book on mobile communications. He serves on the editorial board of the EURASIP Journal on Wireless Communications and Networking.

Dr. Karagiannidis has been a member of Technical Program Committees for several IEEE conferences. He is a member of the editorial boards of the IEEE TRANSACTIONS ON COMMUNICATIONS and the IEEE COMMUNICATIONS LeTters. He is co-recipient of the Best Paper Award of the Wireless Communications Symposium (WCS) in IEEE International Conference on Communications (ICC'07), Glasgow, U.K., June 2007.

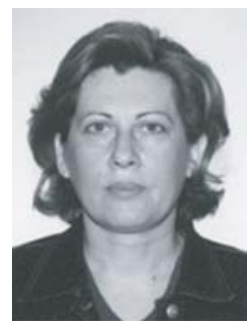

Fotini-Niovi Pavlidou received the $\mathrm{PhD}$ degree and the Diploma in Electrical Engineering from the Aristotle University of Thessaloniki (AUTh), Greece, in 1988 and 1979, respectively. She is with the Department of Electrical and Computer Engineering at AUTh engaged in teaching in the areas of Mobile communications and Telecommunications Networks. Her research interests are in the field of mobile and personal communications, satellite communications, multiple access systems, routing and traffic flow in networks and QoS studies for multimedia applications over the Internet. She is participating in many national and international projects (Tempus, COST, Telematics, IST) and she has been chairing the European COST262 Action on Spread Spectrum Systems and Techniques for Wired and wireless Communications. She has served as member of the TPC in many IEEE/IEE conferences and she has organized/chaired some conferences like, the IST Mobile Summit2002, the Sixth International Symposium on Power Lines Communications-ISPLC2002, the International Conference on Communications-ICT1998, etc. She is a permanent reviewer for many IEEE/IEE journals. She has published more than 100 papers in refereed journals and conferences. She has served as guesteditor for special issues in many journals. She is a senior member of IEEE, currently chairing the joint IEEE VT and AES Chapter in Greece.

of Greece. 